\title{
ATOMIC EMISSION IN THE ULTRAVIOLET NIGHTGLOW
}

\author{
W. E. Sharp and D. E. Siskind
}

Space Physics Research Laboratory, The University of Michigan

\begin{abstract}
An observation of the ultraviolet nightglow between $2670 \AA$ and $3040 \AA$ was conducted over White Sands Missile Range on October 22, 1984, at 0020 hours LST during the Orionids meteor shower. A $1 / 4$-meter uv spectrometer operating at $3.5 \AA$ resolution viewed the Earth's limb at tangent heights between $90 \mathrm{~km}$ and 110 $\mathrm{km}$ for 120 seconds. By inverting the observed limb intensities, a total zenith intensity of $1.4 \mathrm{kR}$ is inferred for the Herzberg I system. Excess emission above the Herzberg I $(7,3)$ band at $2852 \AA$ is identified as the $\mathrm{Mg}$ I resonance line. The intensity ratio of the Herzberg I band system to the $2972 \AA$ line from O $\left({ }^{1} S\right)$ was less than that predicted from the accepted $O\left({ }^{1} S\right)$ branching ratio and acceptable ratios of Herzberg I to $5577 \AA$ emissions. Arguments supporting the identification of the Herzberg III band system are also advanced.
\end{abstract}

\section{Introduction}

The near and middle ultraviolet nightglow results from the recombination of atomic oxygen into molecular oxygen at lower thermospheric altitudes. Initial ground based observation identified the Herzberg I $\left(\mathrm{A}^{3} \Sigma_{\mathrm{u}^{+}}-\mathrm{X}^{3} \Sigma_{\mathrm{g}^{-}}\right)$system (Dufay, 1941) as well as the Chamberlain ( $A^{1}{ }^{3}{ }_{u}-X^{3} \Sigma_{g^{-}}$) system (Chamberlain, 1958). Much later, Slanger and Huestis (1981) identified the presence of Herzberg II ( $c^{1} \Sigma_{\mathrm{u}^{-}}-\mathrm{X}^{3} \Sigma_{\mathrm{g}^{-}}$) emission using synthetic spectra to compare with the ground based data of Broadfoot and Kendall (1968), taken at $5.5 \AA$ resolution. This identification was confirmed when Slanger and Huestis (1983) analyzed some unpublished data taken by Broadfoot at a resolution of $0.5 \AA$. Most recently, Stegman and Murtagh (1988) have presented high resolution (1.3 $\AA$ ) Herzberg I data for 28 nights. Since they also measured the $5577 \AA$ emission concurrently, their data set allows the variability of the Herzberg I to $O\left({ }^{1} S\right)$ ratio to be evaluated.

One limitation of ground based techniques is that observations are limited to near ultraviolet wavelengths longward of the ozone cutoff at $3100 \AA$. For shorter wavelengths, a number of rocket observations have been made; however, due to the low intensity of the airglow, photometers rather than spectrometers are typically used. There is a spectroscopic measurement of the nightglow at wavelengths less than $3100 \AA$ (Hennes, 1966). Although this was a photographic observation at low resolution $(11 \AA)$, he was nonetheless able to identify the Herzberg I band system along with the strong $2972 \AA \mathrm{O}\left({ }^{3} \mathrm{P}-1 \mathrm{~S}\right)$ transition. Degen (1969) was able to quantify the vibrational population of the $A^{3} \Sigma_{u}+$ state from an analysis of that spectrum. At higher resolution, such as the data to be discussed below, it is possible to separate the bandheads of the Herzberg I and II emissions and therefore one can more readily isolate atomic features that would otherwise be masked by the molecular bands. In this note, the O $\left({ }^{3} \mathrm{P}-1 \mathrm{~S}\right)$ transition at $2972 \AA$ is discussed and excess emisson found near $2850 \AA$ is hypothesized to be the resonance line of $\mathrm{Mg}$ I. An identification of the Herzberg III band system is also advanced.

\section{Experiment}

An instrumented payload to study the airglow produced by recombining oxygen atoms was launched from White Sands Missile Range on October 22, 1984 at 0020 LST. This was during the

Copyright 1989 by the American Geophysical Union.

Paper number 89 GL03494.

0094-8276/89/89GL-03494\$03.00 period of the Orionids Meteor shower. It also happened that there was a high level of geomagnetic activity with $A p=47$. The complement of instruments included two resonance lamp modules for measuring atomic oxygen and hydrogen, photometers to measure the emission from the

$\mathrm{NO}_{2}$ continuum, the $\mathrm{OH}(9,3)$ band, and $\mathrm{O}_{2}(0,0)$ Atmospheric Band $(7620 \AA)$, and a $1 / 4$-meter uv scanning spectrometer operating at $3.5 \AA$ resolution. The optical axis of each instrument was aligned along the payload roll axis. The wavelength interval scanned by the digital drive of the spectrometer was $2670 \AA$ to $3040 \AA$. The spectrometer was calibrated in the laboratory with an NBS calibrated tungston lamp. Prior to taking the payload to the launcher, the spectrometer viewed a hollow cathode platinum discharge for the purpose of assigning wavelengths to the data bins. This calibration resulted in the wavelength position of the scan to be accurate to within one grating step $(+/-1.75 \AA)$. The field of view of the telescope was 0.5 by 5.0 degrees.

The payload attitude control system was programmed to do three maneuvers during the flight. First it pointed the payload axis tangent to the trajectory on the upleg after nose cone ejection. Then for 120 seconds centered on apogee the payload was pointed north at a zenith angle of $97^{\circ}$. This placed the field of view for the $90 \mathrm{~km}$ tangent height at $41^{\circ} \mathrm{N}$ latitude (about Boulder, Colorado). While the rocket ascended and descended about apogee, the tangent height changed from $90 \mathrm{~km}$ to $110 \mathrm{~km}$. On the downleg portion of the trajectory the payload axis was pointed tangent to the trajectory facing downward.

\section{Results}

A total of 41 spectral scans were obtained while the payload was viewing the limb. These were averaged together, calibrated and a mean spectrum is displayed as the solid line in Figure 1a. The obvious features are the Herzberg I bands and the $2972 \AA$ line of $O\left({ }^{1} S\right)$. However, to make a comprehensive identification of other features in the data, synthetic spectra were calculated and compared with the data.

The synthetic calculation of the three Herzberg systems used the molecular constants of Slanger and Cosby (1988), the A-values of Degen (1977) and Bates (1989), rotational formulae from Degen (1969) and Kovacs (1969), and an assumed rotational temperature of $200^{\circ} \mathrm{K}$. For the Herzberg III bands, only the $(\Omega=2)$ component of the $A^{3} \Delta_{\mathrm{u}}$ state was considered. The synthetic spectra were fit to the data using a multiple linear regression routine (Bevington, 1962). First, each vibrational level for the $A^{3} \Sigma_{u^{+}}$and the $c^{1} \Sigma_{u^{-}}$ states was separately fit to the data and relative vibrational populations were obtained. It was found, however, that the low counting statistics precluded the determination of vibrational populations for the relatively weak Herzberg II system. Therefore, in the second step of the fitting procedure, the derived vibrational population for the Herzberg I system was used as a guide for a Herzberg II vibrational distribution which was assumed to have the same shape as the Herzberg I but was displaced by 2 quanta to higher vibrational levels (see Slanger and Huestis, 1981). The synthetic spectra were again fit to the data and a revised set of Herzberg I vibrational populations were obtained. In practice, the deduced vibrational distributions obtained from each step were quite similar. The vibrational populations of the Herzberg I system are given in column $A$ of Table 1.

This distribution is in good agreement with that deduced by Degen (1969) from the Hennes (1966) data, with the exception of a reduced $v^{\prime}=8$ population in our spectrum. This value for the $v^{\prime}=8$ population is statistically significant as our fit is guided by the 

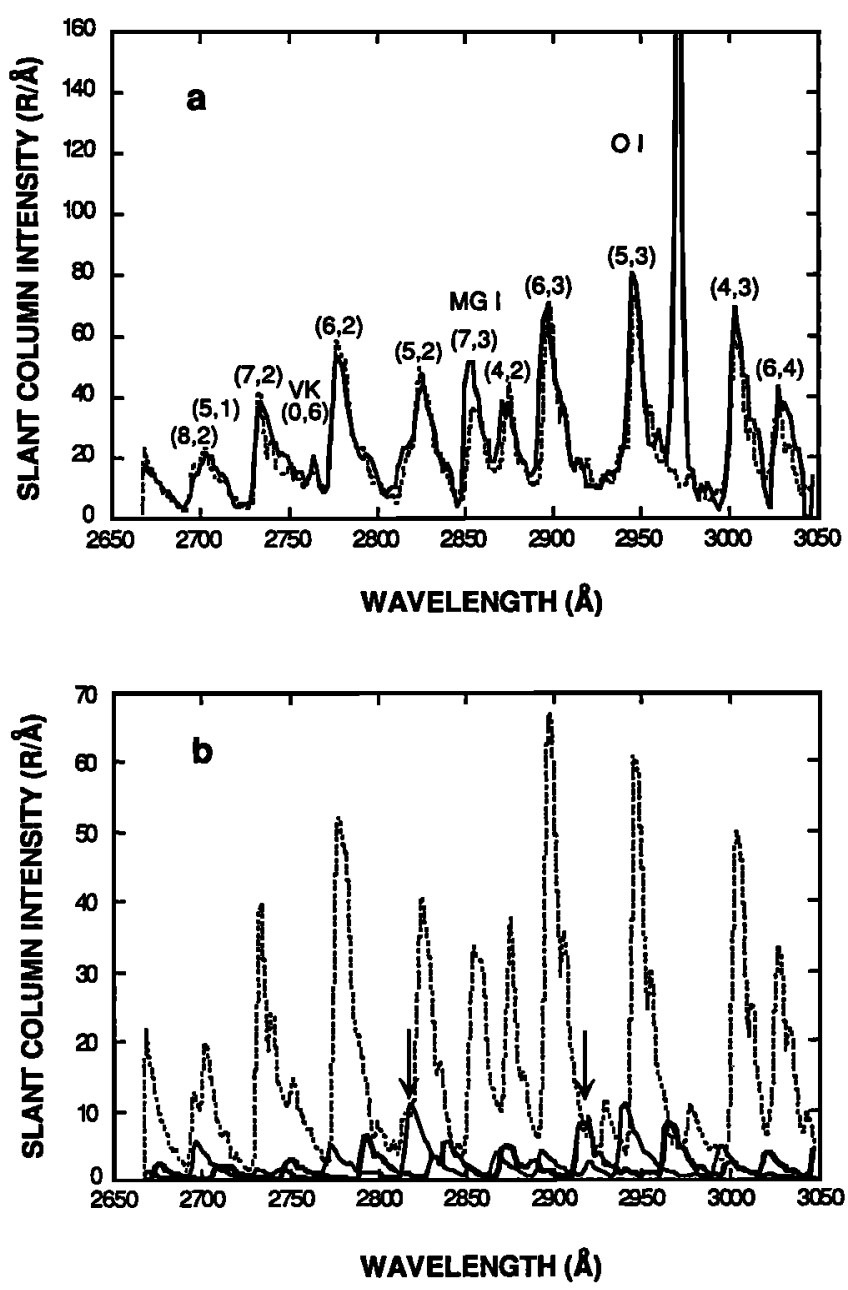

Fig. 1 (a) The data (solid line) obtained from the average of 41 spectral scans obtained while the field of view scanned the limb from approximately $90-110 \mathrm{~km}$. Synthetic spectra for the Herzberg 1 , II, and III and the $\mathrm{N}_{2}$ VK are summed together (dotted line) to compare with the data. (b) The individual synthetic spectra (on expanded vertical scale) for the Herzberg I (dotted), Herzberg II (bold solid line) and Herzberg III (thin solid line) band systems. The two arrows label the Herzberg III $(6,2)$ band at $2815 \AA$ and the Herzberg II $(7,2)$ band at $2913 \AA$.

TABLE 1. Relative Herzberg I Vibrational Populations

\begin{tabular}{lcc}
\hline$v^{\prime}$ & A & B \\
\hline 3 & $0.60+/-.20$ & $0.65+/-.21$ \\
4 & $1.00+/-.08$ & $1.00+/-.09$ \\
5 & $0.84+/-.06$ & $0.91+/-.06$ \\
6 & $0.94+/-.05$ & $0.91+/-.06$ \\
7 & $0.70+/-.04$ & $0.66+/-.04$ \\
8 & $0.18+/-.04$ & $0.16+/-.04$ \\
9 & $0.22+/-.04$ & $0.19+/-.04$
\end{tabular}

\section{A: Populations without contribution from Herzberg III}

B: Populations with contribution from Herzberg III (see text)

relative mix of the $(5,1)$ and $(8,2)$ bands near $2700 \AA$ and our detector is most sensitive at these shorter wavelengths. In addition, our estimate of the intensity in the $(5,1)$ band is constrained by the fit to the relatively bright $(5,2)$ and $(5,3)$ bands.

A search of the data for the previously unobserved Herzberg III band system was made. This was done by constructing spectra using a Herzberg III vibrational distribution patterned after that used by Slanger and Huestis (1981) for the Chamberlain bands; both emissions arise from the same upper state $\left(A^{1}{ }^{3}{ }_{u}\right)$. Inclusion of the the Herzberg III was found to have a small effect on the deduced Herzberg I vibrational populations which are shown in column B of Table 1.

Figure $1 \mathrm{~b}$ is an enlargement of the three Herzberg band systems. From this it is possible to identify regions where various bands predominate. Because many of the Herzberg III bandheads overlap with those of the Herzberg I, the identification of the Herzberg III was not conclusive. However, it should be noted that the short wavelength shoulder of the Herzberg $I(5,2)$ band at $2815 \AA$ does correspond to the location of the Herzberg III $(6,2)$ band, which according to the A-values of Bates (1989) is one of the brightest bands in the Herzberg III system. By contrast, although the fit to the data indicates the Herzberg II to be slightly less bright than the Herzberg III, it is easier to detect because the bandheads are more clearly separated from those of the Herzberg I. Note that while Slanger and Huestis (1981) found the Herzberg II to be 1/4 as bright as the Herzberg I, in this data the Herzberg II are only $1 / 9$ as bright. The most easily identified Herzberg II feature in the spectrum is the $(7,2)$ band at $2913 \AA$.

Other aspects of the fit to the data include the possible presence of $\mathrm{N}_{2}$ Vegard-Kaplan band emission which could result from the observation of a distant auroral arc well to the north and behind the tangent layer. A feature near $2760 \AA$ is identified as the VK $(0,6)$ band. In addition, the strong line at $2972 \AA$ is the $O\left({ }^{3} \mathrm{P}-1_{S}\right)$ line and the excess intensity near $2850 \AA$ is proposed to be due to the resonance line of neutral $\mathrm{Mg}$ which has a wavelength of $2852 \AA$. These features will be discussed below. The combined fit of the Herzberg I, II, III and $\mathrm{N}_{2}$ VK bands is shown as the dotted line in Figure 1a.

Table 2 summarizes the observed emission features and their intensities at the peak of the layer. The Herzberg I intensity is for the total band system based on the fraction observed and the resulting vibrational distribution. The vertical intensity of the Herzberg I was obtained from an "onion-peel" inversion of the altitude profile. Our value of $1.4 \mathrm{kR}$ is brighter than a number of other recent measurements which give total intensities between 230 $700 \mathrm{R}$ for the Herzberg I (see Table 3, to be discussed below). This suggests that the atomic oxygen densities were enhanced on the evening of the measurement. While the $[\mathrm{O}]$ density measurment during the flight failed, we did measure the zenith intensity of the $(0,0)$ band of the $\mathrm{O}_{2}$ atmospheric system $(7620 \AA)$. Our value of 13 $\mathrm{kR}$ for this emission is just larger than the largest value in the range of values recently summarized by Murtagh et al. (1989) and thus provides independent support for our enhanced Herzberg I intensity.

The zenith intensities of the Herzberg II and III systems and the 2972 and $2852 \AA$ lines are deduced from the fraction of those systems required to fit the spectra and the assumption that the layer shapes are the same as the Herzberg I. Because this assumption may not be strictly true, there may be a systematic uncertainty in the derived zenith intensities. On the other hand, the ratio between the various emission features is taken solely from the spectrum shown in Figure 1 and is unlikely to contain significant error. Only the slant intensity of the VK $(0,6)$ band is given since it is a distant emitting surface.

Table 2. Summary of Estimated Emission Rates in Rayleighs

\begin{tabular}{lcc}
\hline Feature & Slant intensity & Zenith Intensity \\
\hline Herzberg I & $70,000+/-2100$ & $1400+/-42$ \\
Herzberg II & $7900+/-1900$ & $158+/-39$ \\
Herzberg III & $8900+/-700$ & $178+/-14$ \\
O' ${ }^{1}$ S) 2972 & $3850+/-790$ & $77+/-16$ \\
Mg I 2852 & $580+/-175$ & $11.5+/-3.5$ \\
Vegard-Kaplan $(0,6)$ & 580 & \\
\hline
\end{tabular}


Table 3: Measurements of Nightglow Intensities (Rayleighs)

\begin{tabular}{llll}
\hline Source & Herzberg I & $5577 \AA$ & Ratio \\
\hline
\end{tabular}

Photometer Measurements:

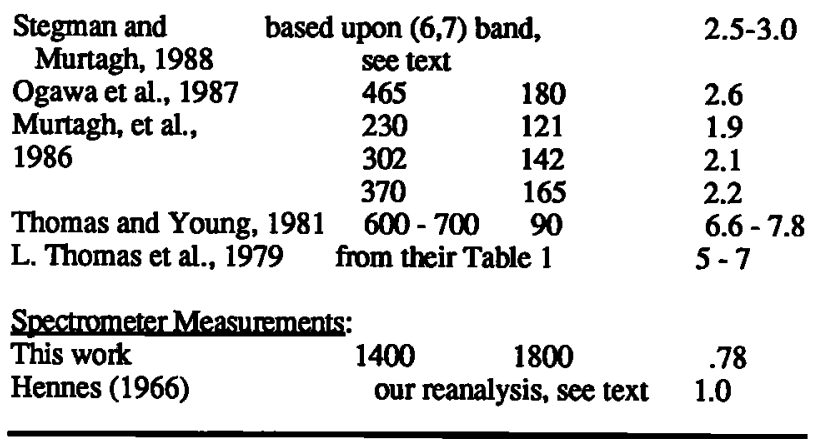

Discussion

\section{2 À Line}

Table 2 shows that the ratio of the intensity of the Herzberg I bands to the $2972 \AA$ is equal to 18 . Using the branching ratio of 23.5 (Kernahan and Pang, 1975) between the $5577 \AA$ and the $2972 \AA$ emissions, we infer a $5577 \AA$ zenith intensity of $1800 \mathrm{R}$. This yields a ratio of Herzberg I to $5577 \AA$ of 0.78 . While we did not simultaneously measure the $5577 \AA$ emission on this rocket flight, there have been numerous other simultaneous measurements of the Herzberg I bands and the $O\left({ }^{1} S\right)$ emission from which the ratio can be deduced. A number of these measurements are presented in Table 3.

Table 3 is divided into two sections. In the first, the 5577 emission was measured with a photometer in conjunction with either a spectroscopic or photometric measurement of the Herzberg I system. In cases where the authors listed the total zenith intensities of both emissions, computation of the ratio was straightforward. For the Stegman and Murtagh (1988) study, they plotted the intensity of the Herzberg $(6,7)$ emission against the green line intensity for 28 separate measurements. Assuming $1.5 \%$ of the total Herzberg emission arises from this single band (Degen, 1977; see also Bates, 1989), we found the ratio to fall within the range 2.5 to 3.0. In general, the mean ratio of Herzberg I to $5577 \AA$ is near 2.5 although Thomas and Young (1981) and L. Thomas et al. (1979) give higher values.

In the second section, the resulting ratio was derived from a simultaneous spectroscopic measurement of both the Herzberg I system and the $2972 \AA$ line where the $5577 \AA$ intensity was deduced using the Kernahan and Pang (1975) branching ratio. At the time of the Hennes (1966) measurement, lack of adequate spectroscopic data precluded a reliable fit to the data. Furthermore, Degen's (1969) analysis of that data did not treat the intensity of the $2972 \AA$ feature. We have reanalyzed that spectrum using the synthetic code applied to this experiment and find that Hennes' (1966) measured intensity ratio agrees with that reported here. The ratio of the Herzberg I to $5577 \AA$ emission derived in this manner is about 1.0. It might be argued that due to the presence of the Vegard-Kaplan feature in the data, contamination by high-altitude auroral emission could be a cause of our discrepancy. We may consider the aurora to be at about a latitude of $50^{\circ} \mathrm{N}$, which is not inconceivable given the geomagnetic activity level. The line of sight to $50{ }^{\circ} \mathrm{N}$ would intersect the aurora at a tangent height of $180 \mathrm{~km}$. Using the ratio of 3 for VK $(0,6)$ to $2972 \AA$ at $180 \mathrm{~km}$ from Sharp $(1970)$ and a slant intensity of $580 \mathrm{R}$ for the VK $(0,6)$ band, an intensity of $193 \mathrm{R}$ is calculated for the slant intensity of $2972 \AA$ which is only $5 \%$ to the signal. An important factor in this discussion is the similarity in the ratio reported here and the Hennes (1966) results. His data were obtained from limb observations also but, they were to the east from White Sands Missile Range where no possibility of auroral contamination exists. Our inferred greenline zenith intensity of 1800 $R$ is significantly greater than previous measurements; the brightest given by Murtagh et al., (1989) is only $400 \mathrm{R}$. We therefore suggest that there is a problem with the branching ratio for $2972 \AA$ from the ${ }^{1} \mathrm{~S}$ state.

Although $2972 \AA$ emission has been measured in a variety of other contexts, such as the terrestrial aurora (Sharp, 1970) and the Venusian dayglow (LeCompte et al. 1989), we note that no simultaneous aeronomic measurements of $2972 \AA$ and $5577 \AA$ exist. Until such a measurement is made and the branching ratio confirmed, the use of the $2972 \AA$ emission to infer the total $O\left({ }^{1} S\right)$ emission could lead to error.

\section{2 Å Line}

Excess emission near $2850 \AA$ is noted in Figure 1a. A comparison of our data with Hennes' (1966) shows that this feature is not present in the earlier spectrum. This can be most clearly seen by noting that in Hennes' spectrum, the $(7,2)$ band at $2732 A$ is slightly brighter than the $(7,3)$ band, while in our data the $(7,3)$ appears significantly brighter. As seen in Figure 1b, both of these features are relatively uncontaminated by other Herzberg bands and since the relative intensities of two bands in a given $v$ ' progression are fixed by the transition probabilities, the most likely explanation for the excess is a contamination by a heretofore unidentified emission.

An expanded view of this feature is shown in Figure 2 along with an estimate of the error in the data due to counting statistics. The excess intensity encompasses about 5-6 wavelength bins or about 9$10 \AA$. This is too narrow in spectral extent to be a molecular feature and thus is most likely an atomic line. Since $\mathrm{Mg}$ is known to be present in the lower thermosphere (Swider, 1984) and since it has a resonance transition at $2852 \AA$, the excess emission is postulated to be the $\mathrm{Mg} I$ resonance line.

Nightglow from metallic atoms (e.g., Na, K) has been previously discussed (e.g. Swider, 1987). There is a transition to the ground state in sodium (5p-3s) at $2852 \AA$, but the exothermicity of the reaction $\mathrm{NaO}$ with $\mathrm{O}$ is not sufficient to produce uv photons. The analogous reaction with magnesium is not exothermic enough to produce a uv photon either. Rocket measurements have shown that $\mathrm{Mg}^{+}$is the dominant metal ion in the nightime lower $\mathrm{E}$ region (Aiken and Goldberg, 1973). Thus an ion-chemical mechanism related to the effective recombination of $\mathrm{Mg}^{+}$is a possible source for the emission.

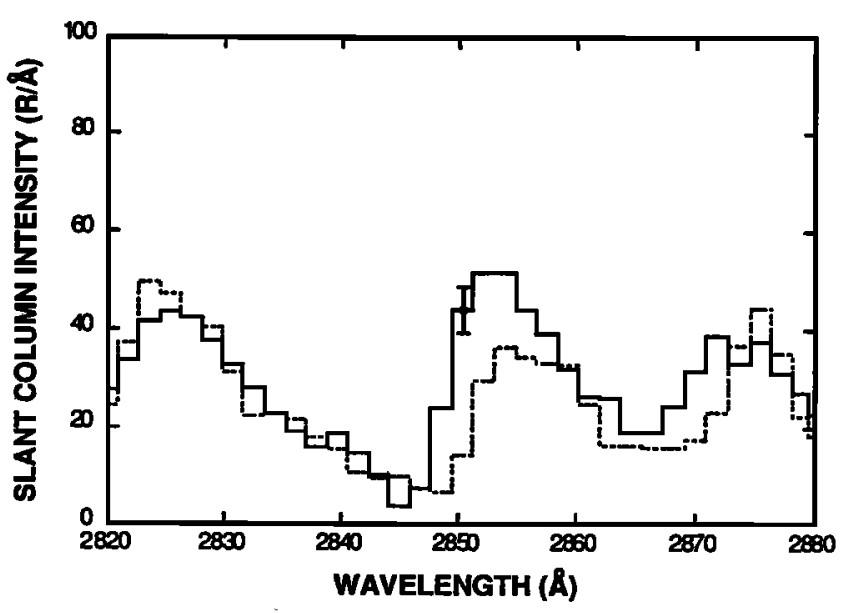

Fig. 2. The wavelength interval around the $\mathrm{Mg}$ emission at 2852 $\AA$. The error bar on the data (solid line) represents the 1 sigma counting statistics. The difference between the synthetic spectra (dashed line) and the data at $2870 \AA$ is not statistically significant as it may result from small errors in the wavelength scale of the instrument. 
Ferguson and Fehsenfeld (1968) present the chemistry of converting $\mathrm{Mg}^{+}$to $\mathrm{Mg}$ through a series of recombinations involving the oxides of the $\mathrm{Mg}$ ion. They deduce an expression for the effective recombination rate which is

$$
\begin{aligned}
\mathrm{K}_{\text {eff }} & =\mathrm{KMgO}_{+}\left[\mathrm{MgO}^{+} / / \mathrm{Mg}^{+}\right] \\
& =7 \mathrm{KMgO}_{+}\left[\mathrm{O}_{3} \mathrm{~V} / \mathrm{O}\right]
\end{aligned}
$$

where the radiative recombination of $\mathrm{Mg}^{+}$is ignored as is the recombination of $\mathrm{MgO}_{2}{ }^{+}$. The rate coefficients measured at $300{ }^{\circ} \mathrm{K}$ (Rowe, et al., 1981) were used. To estimate the densities of $\mathrm{Mg}^{+}$ and $n_{e}$ required to produce the emission, we express the intensity as

$$
4 \pi I=K_{\text {eff }}\left[\mathrm{Mg}^{+}\right]\left[n_{\mathbf{e}}\right] \Delta H
$$

$\Delta \mathbf{H}$ is the half thickness of the emission layer, taken to be about 10 $\mathrm{km}$. From Table 2, the vertical intensity is $11.5 \mathrm{R}$ so that

$$
K_{\text {eff }}\left[\mathrm{Mg}^{+}\right]\left[\mathrm{n}_{\mathbf{e}}\right] \cong 11
$$

Using observed nighttime $\mathrm{O}_{3}$ and $\mathrm{O}$ densities (Hays and Roble, 1973; Sharp, 1980), we estimate Keff to be about $5 \times 10^{-9} \mathrm{~cm}^{3} \mathrm{sec}^{-}$ 1 at $95 \mathrm{~km}$. Given the brightness of the Herzberg emission, the [O] is likely to be greater than that observed by Sharp (1980). If the higher MSIS-86 0 densities are used, the rate decreases to about $2 \times$ $10^{-9} \mathrm{~cm}^{3} \mathrm{sec}^{-1}$. Assuming that the electrons and $\mathrm{Mg}$ ions are equal, one obtains an ion density of $4-7 \times 10^{4} \mathrm{~cm}^{-3}$ for these rate ranges.

Support for this hypothesis comes from an ionosonde measurement made that night from Boulder, Colorado, which indicated the presence of sporadic $\mathrm{E}$. The peak electron density inferred from the signal of $3.3 \mathrm{MHz}$ at $102 \mathrm{~km}$ is $1.5 \times 10^{5} \mathrm{~cm}^{-3}$. As a result, the identification of this emission feature as coming from the ionic recombination of $\mathrm{Mg}^{+}$is the most likely hypothesis. Since this feature does not appear in the Hennes (1966) data, its presence seems to depend upon significant enhancements of the $\mathrm{Mg}$ concentrations, as would be expected during major meteor shower events.

\section{Conclusions}

Data gathered from a mid-latitude rocket borne experiment to measure the middle uv nightglow has identified the presence of the Herzberg I, II, and possibly III emission, an anomalously intense $2972 \AA$ emission from $O{ }^{1}$ S) which raises a question about the branching ratio from $O\left({ }^{1} S\right)$, and excess emission at $2850 \AA$ which is identified to be from $\mathrm{Mg}$ I.

\section{Acknowledgements}

This work was supported by NASA grant NGR-23-005-360. We acknowledge the excellent support provided by the NASA Wallops Flight Facility team led by David Detwiler.

\section{References}

Aiken, A .C. and R. A. Goldberg, Metallic ions in the equatorial ionosphere, J. Geophys. Res., 78, 734, 1973.

Bates, D. R., Oxygen band system transition arrays, Planet. Space Sci., 37, 881, 1989.

Bevington, P.R., Data Reduction and Error Analysis for the Physical Sciences, McGraw-Hill Book Company, 1962, 336pp.

Broadfoot, A. L. and K. R. Kendall, The airglow spectrum, 3100 - 10000 \&, J. Geophys. Res.,73, 426, 1968.

Chamberlain, R. W., The blue airglow spectrum., Astrophys. J., 128, 713, 1958.

Degen, V., Vibrational populations of $\mathrm{O}_{2}\left(\mathrm{~A}^{3} \Sigma_{\mathrm{u}}{ }^{+}\right)$and synthetic spectra of the Herzberg bands in the nightglow, J. Geophys. Res., 74, 5145, 1969.

Degen, V., Nightglow emission rates in the $\mathrm{O}_{2}$ Herzberg bands, J. Geophys. Res., 82, 2437, 1977.
Dufay, J., Une intepretation possible de certaines radiaions intenses du ciel nocturne dans la region ultraviolette., Compt. Rend. Acad. Sci. Paris , 213, 284, 1941.

Ferguson, E. E. and F. C. Fehsenfeld, Some aspects of the metal ion chemistry of the earth's atmosphere, J. Geophys, Res., 73, 6215, 1968.

Hays, P. B. and R. G. Roble, Observation of mesospheric ozone at low latitudes, Planet Space Sci., 21, 273, 1973.

Hennes, J. P., Measurement of the ultraviolet nightglow spectrum, J. Geophys. Res., 71, 763, 1966.

Kemahan,. J.A. and P.H.L. Pang, Experimental determination of absolute A coefficients for "forbidden" atomic oxygen lines, Can. J. Phys., 53, 455, 1975.

Kovacs, 1., Rotational Structure in the Spectra of Diatomic Molecules, American Elsevier, Publishing Co., 1969, 320 pp.

LeCompte, M. A., L. J. Paxton, and A. I. F. Stewart, Analysis and interpretation of observations of airglow at $297 \mathrm{~nm}$ in the Venus thermosphere, J. Geophys. Res., 94, 208, 1989.

Murtagh, D. P., I. C. McDade, R. G. H. Greer, J. Stegman, G. Witt, and E. J. Llewellyn, ETON 4: an experimental investigation of the altitude dependence of the $\mathrm{O}_{2}\left(\mathrm{~A}^{3} \Sigma_{\mathrm{u}}{ }^{+}\right)$ vibrational populations in the nightglow, Planet. Space Sci., 34, 811, 1986.

Murtagh, D. P., G. Witt, J. Stegman, I.C. McDade, E. J. Llewellyn, F. Harris, and R.G.H. Greer, An assessment of proposed $O\left({ }^{1} S\right)$ and $\mathrm{O}_{2}\left(\mathrm{~b}^{1} \Sigma_{\mathrm{g}}{ }^{+}\right)$nightglow excitation parameters, Planet. Space Sci., 1989, in press.

Ogawa, T., N. Iwagami, M. Nakamura, M. Takano, H. Tanabe, A. Takechi, A. Miyashita, and K. Suzuki, A simultaneous observation of the height profiles of the night airglow of OI $5577 \AA, \mathrm{O}_{2}$ Herzberg and Atmospheric bands, J. Geomug. Geoelectr. 39, 211, 1987.

Rowe, B. R., D. W. Fahey, E. E. Ferguson, and F. C. Fesenfeld, Flowing afterglow studies of gas phase magnesium ion chemistry, J. Chem. Phys. 75, 3325, 1981.

Sharp, W. E., Rocket-bome measurements of the nitrogen Vegard-Kaplan bands in the ultraviolet aurora, Ph .D. Thesis, University of Colorado, 1970.

Sharp, W. E., Absolute concentrations of $O\left({ }^{3} \mathrm{P}\right)$ in the lower thermosphere at night, Geophys. Res. Lett., 7,485, 1980.

Slanger, T. G. and D. L. Huestis, $\mathrm{O}_{2}\left(\mathrm{c}^{1} \Sigma_{\mathrm{g}}^{-}-\mathrm{X}^{3} \Sigma^{-} \mathrm{g}\right)$ emission in the terrestrial nightglow, J. Geophys. Res., 86, $3551,1981$.

Slanger, T. G. and D. L. Huestis, The rotationally resolved 3400 to $3800 \AA$ terrestrial nightglow, J. Geophys. Res., 88, 4137, 1983.

Slanger, T. G. and P.C.Cosby, $\mathrm{O}_{2}$ Spectroscopy below 5.1 eV, J. Phys. Chem., 92, 267, 1988.

Stegman, J. and D. P. Murtagh, High resolution spectroscopy of oxygen U.V. airglow, Planet Space Sci., 36, 927, 1988.

Swider, W., Ionic and neutral concentrations of $\mathrm{Mg}$ and $\mathrm{Fe}$ near 92 km, Planet. Space Sci., 32, 307, 1984.

Swider, W., Chemistry of mesospheric potassium and its different seasonal behavior as compared to sodium, J. Geophys. Res., 92, 5621, 1987.

Thomas, L., R. G. H. Greer, and P. H. G. Dickinson, The excitation of the $557.7 \mathrm{~nm}$ line and Herzberg bands in the nightglow, Planet. Space Sci., 27, 925, 1979.

Thomas, R.J. and R. A. Young, Measurement of atomic oxygen and related airglows in the lower thermosphere., J. Geophys. Res., 86, 7389, 1981.

W. E. Sharp and D. E. Siskind, Space Physics Research Laboratory, Department of Atmospheric, Oceanic and Space Sciences, The University of Michigan, Ann Arbor, MI 48109 2143.

(Received: October 10, 1989; revised: November 8,1989 ; accepted: November 13, 1989) 